\title{
Giacomo Puccini (1858-1924): un malato di musica e di altro
}

\section{Luca Berni}

\author{
Direttore della programmazione di Rete Toscana Classica, Firenze
}

\begin{abstract}
Giacomo PuCCini (1858-1924): CRAZy ABOUt MUSiC AND MORE
Abstract. Giacomo Puccini was born in Lucca on December 22, 1858, the sixth of nine children of Michele Puccini and Albina Magi, and the last member of an 18th century pedigree of musicians. Heir of Giuseppe Verdi's melodramatic tradition, Puccini had enormous success on the European and American scenes. He was a passionate hunter but also liked cars, cigarettes, cigars, motorboats and buying and restoring houses. A car crash in 1903 resulted in a poorly healing tibial injury; he was subsequently diagnosed with diabetes and started taking saccharin but in 1923 initiated insulin therapy at empiric dosages. After accidentally swallowing a goose bone in 1923, he began to suffer from recurrent laryngitis, earaches, swallowing pain, dysphonia and swelling of the neck so severe that he could not close his shirt collar. Like after the car crash, famous physicians were consulted. On November 3, 1924, a collegial consultation in Florence among professors Torrigiani, Toti and Gradenigo referred him to doctor Louis Ledoux in Bruxelles, who used radiation therapy for laryngeal cancer. Puccini died on November 29, 1924 in Bruxelles, leaving behind an incomplete Turandot that had its stage debut on April 25, 1926, conducted by Arturo Toscanini.
\end{abstract}

Key words: Puccini, Disease, Cars, Tibia, Diabetes, Cancer, Larynx, Radium

Conflict of interest: None.

Financial support: None.

Accettato: 26 Maggio 2014

Giacomo Antonio Domenico Michele Secondo Maria Puccini è nato a Lucca il 22 dicembre 1858 ed è morto a Bruxelles il 29 novembre 1924, segno Capricorno, era il sesto di nove figli di Michele Puccini e Albina Magi e l'ultimo rappresentante di una famiglia di musicisti, una delle linee generazionali più lunghe della storia della musica dopo i Bach e i Couperin. I nomi: Giacomo in ricordo del trisnonno, Antonio del bisnonno, Domenico del nonno e Michele del padre racchiudono un vero e proprio spaccato della Lucca musicale dal XVIII secolo al XX. Anche il fratello di Puccini, Michele, il nono figlio nato dopo la morte prematura del padre, tenterà la sorte come musicista, ma emigrato in Sudamerica morirà nel 1891 a soli 27 anni.

Le sorelle di Puccini che qui conviene nominare anche per il fatto della estrema particolarità dei nomi di battesimo: Otilia, Tomaide, Temi, Nitteti, Iginia (Suor Giulia Enrichetta), Ramelde e Macrina, non erano musiciste di professione, ma avevano dimestichezza con la musica che si può affermare fosse il nutrimento della famiglia.

Di tutta la dinastia Puccini Giacomo fu il compositore più fortunato e di rilievo infatti dopo Giuseppe Verdi fu proprio
Puccini a tenere alte le sorti dell'opera italiana nel mondo e con risultati che si possono considerare di assoluta completezza musicale.

Credo sia importante, ai fini anamnestici, leggere il ritratto di Puccini che un amico di lunga data, il pittore Ferruccio Pagni, ci consegna nelle pagine di un libro scritto da Guido Marotti, Giacomo Puccini intimo, pubblicato da Vallecchi nel 1926:

Testa ben modellata, fronte spaziosa e serena, mascella soda, mento allungato e squadrato, bocca piccola, sensuale, gl'incisivi superiori sporgenti, naso prominente - era proprio il perfetto tipo toscano, forte, vigoroso, con due occhi grigio-marroni, non grandi - la palpebra sinistra lievemente abbassata - a vivaci fino alla più raffinata furbizie, malinconici, a volte, fino alla tristezza più accorata (Fig. 1). Aveva una maschera mutevole e alcune asimmetrie gli donavano una speciale caratteristica, accentuatasi con l'andare degli anni. Da giovane, i capelli castano-scuri erano ricciuti, a larghe anella, e i baffi, più grossi e forti di quelli americanizzati negli ultimi anni. Quando lo conobbi, la figura, più che tarchiata, minacciava corpulenza; poi, con l'invecchiare, il 


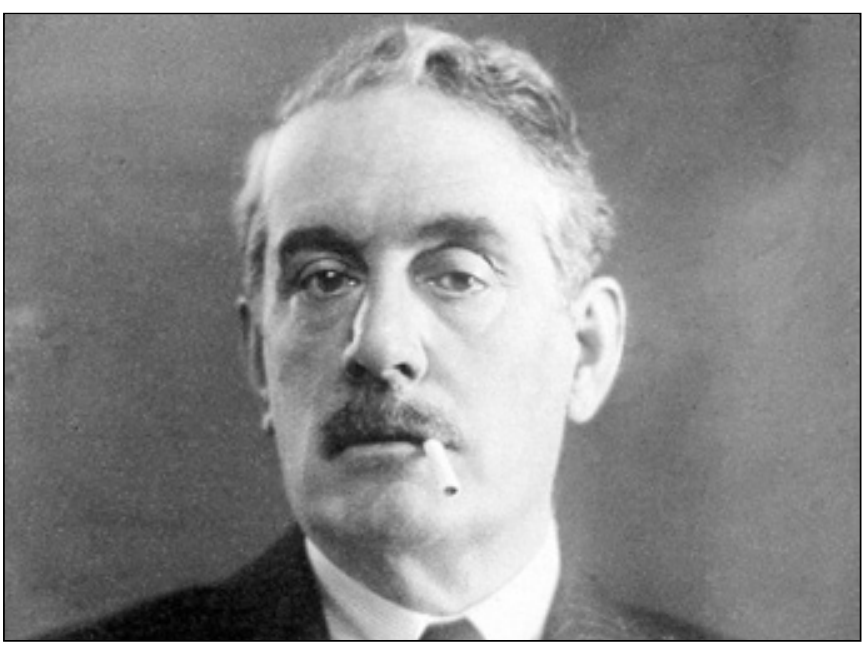

Fig. 1 - Giacomo Puccini negli anni ‘20.

maestro è andato affinandosi nei lineamenti e nella sagoma della persona. La sua voce baritonale si velava facilmente (Puccini è sempre stato accanito fumatore di pipa, di sigari, di sigarette) e quando cantava aveva il registro di tenore e un ottimo "falsetto» col quale deliziosamente accennava e parti di donna.

Oltre alla musica, Puccini ha amato la caccia, le automobili, le sigarette, i sigari, i motoscafi, e le abitazioni da acquistare e quindi ristrutturare.

La caccia era stato il movente per cui si trasferì a Torre del lago, dove poi costruì la villa, oggi museo e dove poteva sfogare il talento di cacciatore agli acquatici sia in maniera legale che di frodo che, per il suo temperamento, era quasi da preferirsi. Quando il marchese Carlo Ginori Lisci gli accordò il permesso di cacciare, Puccini per sdebitarsi gli dedicò $L a$ bohème e scrisse una lirica per voce e pianoforte su versi di Renato Fucini, Avanti Urania! per il varo del suo yacht a vapore.

Nella notte tra il 25 e il 26 febbraio del 1903 mentre tornava da Lucca a Torre del lago a bordo della Clément Bayard, guidata dall'autista Guido Barsuglia, all'altezza di Vignola di San Macario l'automobile esce di strada e cade nel fosso, Elvira e il figlio Antonio sono illesi, l'autista, sbalzato fuori dall'abitacolo si frattura il femore sinistro, ma chi ha la peggio è il maestro che, rimasto incastrato sotto la vettura a contatto con i vapori della benzina è semisvenuto e riporta una frattura della tibia destra con spostamento e travaso. Soccorso da un medico locale il dottor Sbragia, viene portato a Torre del lago col barcone di soccorso del marchese Ginori. Da questo momento la guarigione del compositore sarà lunga e complessa, medici illustri si alternano al capezzale del paziente. La medicazione provvisoria viene tolta l'11 marzo e il 14 marzo gli viene applicata un'armatura che immobilizza la gamba provocando fortissimi dolori al paziente che passano molto lentamente. Il 12 maggio viene tolto l'apparecchio, ma la calcificazione dell'osso era incompleta, in una lettera al librettista Luigi Illica scrive che gli hanno

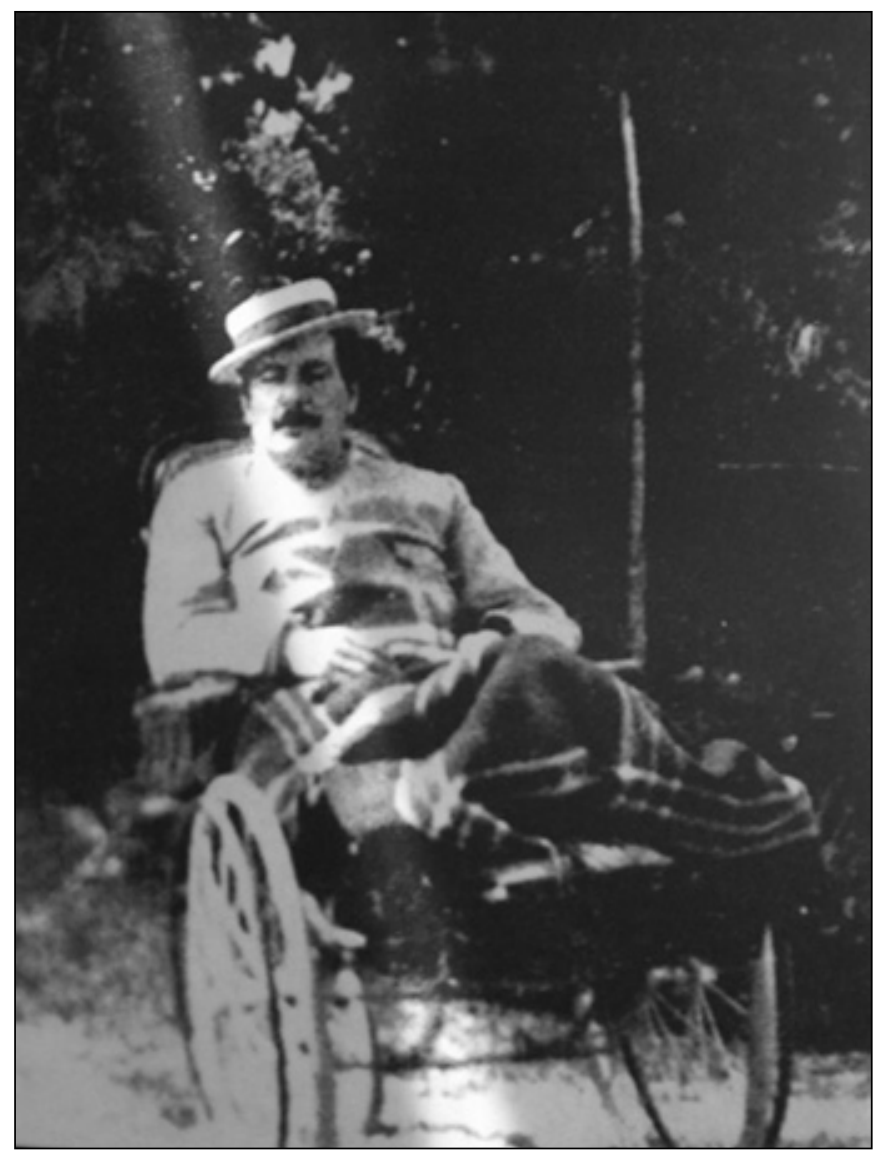

Fig. 2 - Giacomo Puccini convalescente dopo l'incidente automobilistico del 1903.

ordinato delle punture di fosfati e stricnina. Il 20 giugno arrivano a consulto da Genova i professori Guarnieri e Novaro che prevedono che in un mese Puccini avrebbe camminato di nuovo. Alla sorella Ramelde il 25 giugno 1903 scrive: Il consulto fu confortante. Dice Novaro che camminerò tra un mese! Intento mi farà... un'armatura d'acciaro... aro, dalla 'oscia alle nocche del malleolo. Sarà vero? 1000 lire, 1000 lire, 1000 lire m'ha preso Novaro. Sono affranto! Nella ridda di luminari Puccini ad agosto si fa visitare anche dall'esimio ortopedico bolognese Alessandro Codivilla, direttore dell'Istituto Ortopedico Rizzoli di Bologna, ma le ossa (gli ossi come vengono chiamati nella corrispondenza) sono sempre mobili. Il 30 giugno 1903 la guarigione nonostante le assicurazioni dei medici è ancora molto lunga: Ho lascito le grucce a vado con i bastoni ma molto male... l'apparecchio l'ho sempre scrive alla sorella Ramelde. Essendogli stato diagnosticato il diabete altro che all'inizio del 1904 non si poterono valutare con precisione i danni renali che, determinando un cattivo metabolismo, furono la causa della mineralizzazione dell'osso (Fig.2).

In una lettera all'amico Luigi Pieri del marzo 1903 in cui Puccini descrive con ironico sorriso la vita di ammalato $\mathrm{cu}-$ rato dalla moglie, dalla sorella di lei Ida e dall'amata sorella Nitteti: il sollevamento della gamba, i cuscini, la posizione nel letto, il dolore provato, ma anche cinque pasti abbondanti 


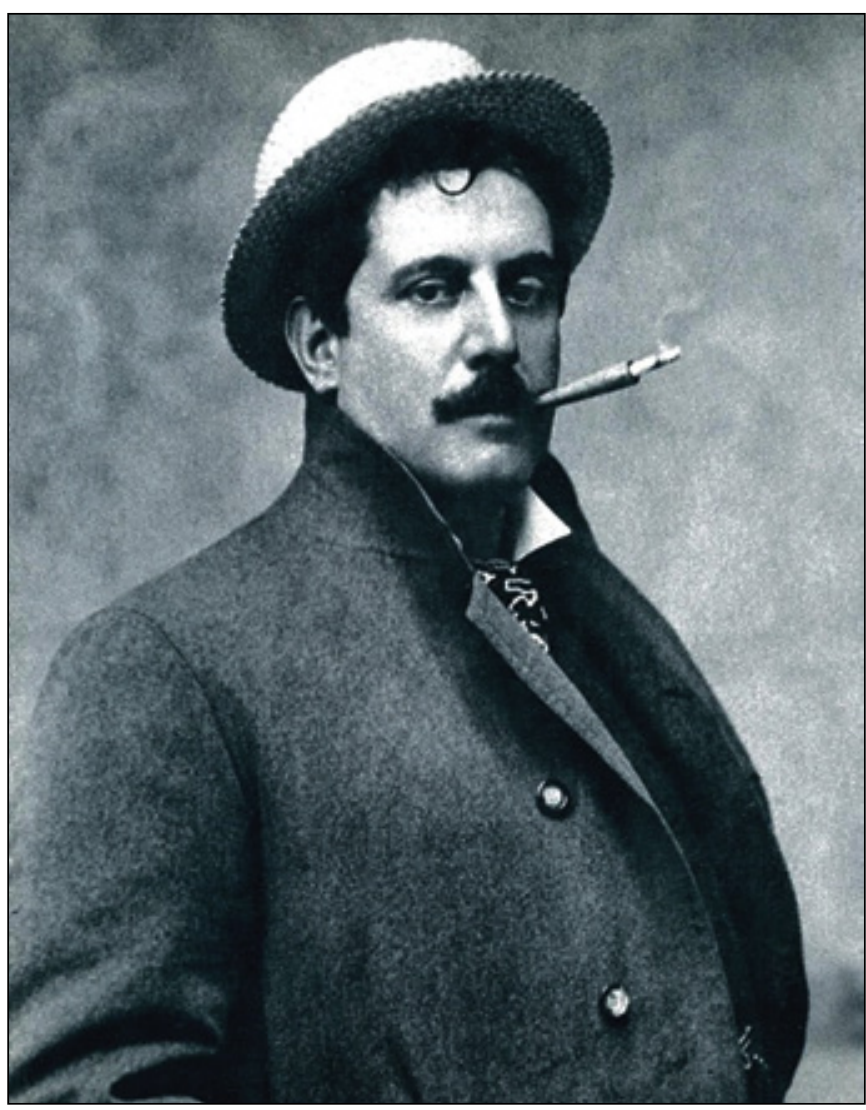

Fig. 3 - Giacomo Puccini giovane di belle speranze e grande fumatore.

e una trentina di sigarette oltre alle lettere, ai telegrammi e alle visite degli amici più prossimi. L'editore milanese Tito Ricordi alla fine dell'articolo - pubblicato sulla Gazzetta musicale di Milano, organo ufficiale delle Edizioni Ricordi - intitolato Catastrofe automobilistica del maestro Giacomo Puccini (articolo corredato anche dalle foto prese da un amico di Puccini Alfredo Caselli), scrive: Trovato Puccini in ottime condizioni generali, di spirito sollevato e quasi col solito umore faceto [...]. Commosso per tante e tante prove di affetto e di interessamento, Puccini ringrazia vivamente e cordialmente. Puccini nonostante la frattura a un certo momento riprese anche a comporre Butterfly al pianoforte a coda che può avvicinare con la sedia a rotelle e la gamba distesa.

La passione per le auto, che tra il 1901 e il 1924 furono tredici otre a una Harley Davidson con sidecar, a quattro motoscafi e uno yacht, dette modo ai medici di diagnosticare il diabete a Puccini che dal 1904 usa la saccarina e alla sorella Ramelde scrive: Il piscio è buono non buonissimo però... alludendo alla pratica della degustazione dell'urina per determinarne la presenza di glucosio. Puccini è stato un grande fumatore di sigarette e sigari fino da giovanissima età, (Fig.3) la leggenda vuole che avesse rubato le canne dell'organo, di cui era titolare, della chiesa di San Pietro in Somaldi per procurarsi da fumare. Nel 1908 durante un viaggio in Egitto si fece preparare due miscele di tabacchi speciali che poi si faceva regolarmente inviare in Italia sia per se che per la moglie, altrettanto accanita fumatrice. Inutile dire che le sigarette si chiamavano... «Puccini». Già a 25 anni, nel 1883, anno del anno del diploma in composizione, in una lettera alla madre scrive dopo la resezione di un ascesso in gola: Sono stato molto male nei giorni passati ma sto meglio dopo il taglio in gola. Domani o domani l'altro m'alzerò dal letto, sono un po'dimagrito. Ho sofferto assai per dolori di testa terribile. Ho dovuto tener ghiaccio in testa e alla gola. Per sovrappiù avevo un po' di riscaldamento intestinale che mi metteva un po'di paura...

Nell'epistolario con Riccardo Schnabl (Giacomo Puccini. Lettere a Riccardo Schnabl, Emme Edizioni, Milano 1981), un ricco amico italo austriaco che lo ragguagliava sulle novità in ambito operistico da tutta Europa, troviamo un'indicazione terapeutica di discreta importanza: Farò la cura dell'Insulin il celebre farmaco di Toronto per il diabete - te ne dirò - l'ho potuto avere da Londra in riguardo speciale. Per Puccini 1'Insulin (sperimentata con successo nel 1922 per la cura del diabete) era l'alternativa alla cura di Steinach cui si apprestava a sottoporsi: celebre cura per il ringiovanimento cellulare. Si trattava per Puccini non tanto di una soluzione al tasso glicemico, ma quasi di una curiosità farmacologica che gli arriva per riguardo speciale. Curava il diabete, con indicazioni terapeutiche molto probabilmente rudimentali ed empiriche. Il diabete da lui - e probabilmente dai suoi medici - non fu tenuto mai nella giusta considerazione e lo dimostra la debilitazione già riscontrata nella difficile calcificazione della tibia dopo l'incidente automobilistico. Nell'estate del 1923 a Ingolstad a Puccini fu estratto un osso d'oca dall'ipofaringe, a questo incidente seguì un periodo di forti mal di gola, sempre a Riccardo Schnabl scrive: Il mio mal di gola mi dà gran noia - ho visto, come t'ho scritto già 4 specialisti - chi mi consiglia una cura chi un'altra. Penso che in Germania e in Svizzera ci debbano essere specialisti bravi - Tu ne conosci? informati. Il mio male è Faringite che da 7 mesi mi tormenta - scrivimi subito - ciao ti abbraccio tuo Giacomo. Al momento in cui il mal di gola si fece più insistente con otalgia e dolori alla deglutizione, peggiorando la disfonia e si manifestarono tumefazioni cervicali tali da non permettergli di chiudere il colletto della camicia. Puccini allora decise di farsi visitare da un celebre otorinolaringoiatra di Firenze: il professor Camillo Arturo Torrigiani dal quale si recò per un consulto. Si trattava di un papilloma sotto l'epiglottide alla radice della lingua delle dimensioni di una noce. Torrigiani era in contatto con il professor Moss di Berlino che utilizzava il radium per trattare il cancro della faringe, ma Moss si era ritirato dalla professione. Ci furono poi due ulteriori consulti: con il professor Toti di Firenze (che al figlio Antonio disse che il cancro era di natura gravissima) e con il professor Gradenigo di Napoli. Il 3 novembre 1924 un consulto generale con Torrigiani, Toti e Gradenigo a Firenze, consigliò al compositore di recarsi nella clinica del dottor Louis Ledoux di Bruxelles che eseguiva un trattamento radio-chirurgico per i tumori della laringe. Al librettista Giuseppe Adami Puccini scrisse: ... Sono in un periodo tremendo. Questo mio mal di gola mi tormenta, ma più moralmente che per la pena fisica. Andrò a Bruxelles da 
un celebre specialista. Partirò presto. Mi opererò? Mi si curerà? Mi si condannerà? Così non posso più andare avanti. e Turandot è li. Al ritorno da Bruxelles mi metterò al lavoro. Pochi giorni prima di partire per Bruxelles Puccini aveva incontrato Arturo Toscanini, con cui erano sorti degli screzi. Toscanini avrebbe dovuto dirigere la prima di Turandot e con lui Puccini aveva discusso piani per la prima dell'opera, la distribuzione dei ruoli, i bozzetti, la messa in scena, e soprattutto quello che a Puccini premeva di più per le grandi difficoltà che stava incontrando: il finale dell'opera.

Puccini partì in treno per Bruxelles il 4 novembre con il figlio Antonio e con Clausetti (l'amministratore di Casa Ricordi). Durante il viaggio per non impressionare i compagni di viaggio per ogni colpo di tosse accompagnato da sanguinamento Puccini gettò i fazzoletti dal finestrino. Il 5 novembre si sottopose alla prima visita del professor Ledoux al Radium Institute in Avenue de la Couronne che, dopo aver eseguito una biopsia faringea oltre agli esami di rito al figlio non dette speranze escludendo una operazione chirurgica e proponendo quindi un trattamento di tracheotomia, svuotamento laterocervicale con applicazioni di radio e infine applicazione inserimento di aghi radioattivi direttamente sul tumore. Tre tempi distanziati da dieci giorni per permettere al fisico di Puccini di riprendersi. A causa del diabete si escluse anche lo svuotamento laterocervicale per iniziare il trattamento con il collare contenente radium. La tracheotomia per permettere di respirare e il collare spaventarono molto Puccini che nelle lettere si raccomanda di non rivelare i particolari della cura alla moglie.

La prima fase con il collare di radium fu relativamente facile: si trattava di applicare il collare per alcune ore al giorno. Poi la vita del musicista scorreva in maniera normale, al Théâtre de la Monnaie si dava Butterfly e vi assistette: riconosciuto dal pubblico fu applaudito con calore. Ricevette visite e le condizioni fisiche migliorarono con la cessazione dell'emofotoe che sollevò il paziente al punto di indurlo a ricominciare a fumare. Arrivarono, chiamati dal figlio, Riccardo Schnabl, Sybil Seligman (carissima amica londinese del maestro), Angelo Magrini (industriale toscano) e la figlia

\footnotetext{
${ }^{1}$ Ultime notizie «Il Messaggero toscano» del 4 dicembre 1924 - Pisa. Le onoranze funebri al m.o Puccini. Le solenni esequie nel Duomo di Milano. Alle 6.30 del 3 dicembre la salma di Giacomo Puccini era stata trasferita dalla Chiesa di S. Fedele al catafalco eretto nel centro del coro senatoriale del Duomo. Viole e garofani rossi sul feretro foderato dal tricolore. Davanti, a sinistra, la corona del re d'Italia e a destra quella del re del Belgio. Appoggiate sui lati quelle di Mussolini, del fascio italiano di Bruxelles e di Albertini-Giacosa, e in linea quelle dei famigliari. Ai fianchi del feretro erano in servizio d'onore quattro valletti in nero e argento e quattro vigili comunali. Alle pareti, sugli organi e sui pulpiti grandi drappeggi di velluto nero a frange d'oro. Sulla facciata campeggiava un'epigrafe «Lacrime e preci per Giacomo Puccini dalla gloria terrena asceso alla gloria del cielo». Messa funebre ed ossequio di una immensa folla. Benito Mussolini aveva pronunciato un discorso, e Toscanini aveva diretto la triste musica della messa di Requiem dell'Edgar.
}

di primo letto di Elvira, Fosca, adorata dal maestro che la considerò sempre come una figlia.

La seconda fase della cura ebbe inizio il 22 o il 24 novembre con una seduta chirurgica di quasi quattro ore durante la quale, in anestesia locale, la laringe fu irradiata direttamente a livello del tumore con una terapia radiologica da contatto. Tuttavia durante l'intervento si ebbe un forte sanguinamento che impedì la chiusura della ferita e fu introdotto nel naso un sondino naso-gastrico. Durante questa fase Puccini non poté parlare altro che tramite biglietti, che in gran parte sono conservati e che sono una terribile testimonianza del momento che stava vivendo: Mi sento come se avessi delle baionette in gola - Sto peggio di jeri l'inferno in gola e mi sento svanire, acqua fresca, fino allo straziante estremo biglietto con cui evoca Elvira povera donna finita.

Furono cinque giorni difficili, animati anche da cauto ottimismo, ma la sera del 28 novembre la fibra del musicista cede: ha un attacco cardiaco e perde conoscenza. Non serve il pronto intervento di Ledoux che rimuove gli aghi dalla laringe, alle 11.30 del 29 novembre Puccini muore dopo aver ricevuto l'estrema unzione dal monsignor Micara Nunzio Apostolico in Belgio. Puccini il $1^{\circ}$ dicembre fu onorato da una cerimonia funebre a Bruxelles nella chiesa di Sainte Marie officiata da monsignor Micara. Il feretro fu poi accompagnato alla stazione ferroviaria e posto in un vagone speciale che lo condusse a Milano. Il 3 dicembre $^{1}$ si svolsero a Milano in Duomo i funerali ufficiali quindi la salma fu tumulata provvisoriamente nella cappella Toscanini al cimitero Monumentale.

Giacomo Puccini dal 1925 riposa nella villa di Torre del lago in una cappella voluta dal figlio Antonio che commissionò la decorazione funebre ad Antonio Maraini.

Turandot rimase senza finale. Fu eseguita alla Scala il 25 aprile 1926 e, nonostante che fosse stato commissionato a Franco Alfano un finale sugli appunti di Puccini, Toscanini depose la bacchetta alla morte di Liù dicendo: Qui finisce l'opera perché a questo punto il maestro è morto. Turandot. Nonostante l'improba fatica di Alfano di scriverne finale o la recente scrittura Luciano Berio, Turandot è un'opera incompiuta e dovrebbe essere rappresentata come tale. Nel manoscritto dopo la morte di Liù, Puccini scrive poi Tristano, allusione sibillina che allude al capolavoro di Wagner, che ci dice che forse non gli era possibile scrivere un finale tradizionale e che, forse per le questioni di salute o per un blocco creativo, era meglio lasciare sospeso: un momento di riflessione che forse si sarebbe risolto, ma che sarebbe doveroso lasciare incompleto.

\section{Riassunto}

Giacomo Puccini, nato a Lucca il 22 dicembre 1858, era il sesto di nove figli di Michele Puccini e Albina Magi e l'ultimo rappresentante di una genealogia di musicisti risalente al XVIII secolo. Erede della tradizione melodrammatica verdiana ebbe un enorme successo in vita in tutti $i$ teatri d'Europa e in America. Appassionato cacciatore, altre sue passioni furono: le automobili, le sigarette, i sigari, i motoscafi, e le abitazioni da acquistare e ristrutturare. 
Un'incidente d'auto nel 1903 e la difficile calcificazione della tibia destra portarono alla diagnosi di un diabete e all'uso della saccarina. Nel 1923 quasi per caso inizia a curarsi con l'Insulin, ma senza i dosaggi e le tecniche di cura oggi conosciute. Sempre nel 1923 ingoia un osso d'oca che gli deve essere estratto, da allora, fumatore accanito di sigari sigarette, accusa insistenti laringiti con otalgie fino a dolori alla deglutizione, disfonia e infine tumefazioni cervicali tali da non permettergli di chiudere il colletto della camicia. Furono interpellati illustri medici del tempo, come era accaduto per l'incidente d'auto. Il 3 novembre 1924 un consulto generale con i professori Torrigiani, Toti e Gradenigo a Firenze, consigliò al compositore di recarsi nella clinica del dottor Louis Ledoux di Bruxelles che eseguiva un trattamento radio-chirurgico per i tumori della laringe. Puccini morì a Bruxelles il 29 novembre 1924 lasciando incompiuta Turandot che sarà rappresentata postuma, con la direzione di Arturo Toscanini il 25 aprile 1926.
Parole chiave: Puccini, Malattie, Automobili, Tibia, Diabete, Cancro, Laringe, Radium

Dichiarazione di conflitto di interessi: L'Autore dichiara di non avere conflitto di interessi.

Contributi economici agli Autori: L'Autore dichiara di non aver ricevuto sponsorizzazioni economiche per la preparazione dell'articolo.

\section{Indirizzo degli Autori:}

Luca Berni

Direttore della programmazione di Rete Toscana Classica,

Firenze

lucaberni@dada.it

programmazione@retetoscanaclassica.it

(www.retetoscanaclassica.it)

\section{Letture, siti, video e ascolti consigliati}

La voce di Puccini registrata a New York nel 1907:

https://www.youtube.com/watch?v=SPXPZWilkAk\&list=PL CF3CEFF91B20696F\&index $=2$

\section{Biografie}

Julian Budden, Puccini, Carocci, Roma 2002

Michele Girardi, Giacomo Puccini. L'arte internazionale di un musicista italiano, Marsilio, Venezia 1995.

\section{Qualche ascolto selezionato}

Tosca: direttore Victor De Sabata/EMI

La Bohème: direttore Herbert von Karajan/Decca

Suor Angelica: direttore Bruno Bartoletti/RCA

Fanciulla del West: direttore Zubin Mehta/DGG

Crisantemi per quartetto d'archi: Quartetto Hagen/DGG

\section{Siti}

www.puccini.it (Centro Studi Giacomo Puccini)

www.puccinimuseum.org (Casa natale di Puccini a Lucca) 